\title{
Isorhamnetin protects against bleomycin-induced pulmonary fibrosis by inhibiting endoplasmic reticulum stress and epithelial-mesenchymal transition
}

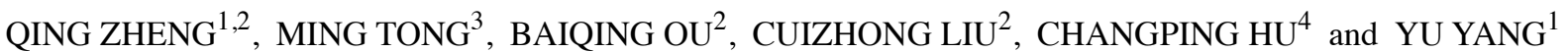 \\ ${ }^{1}$ Department of Geriatrics, The Second Xiangya Hospital of Central South University, Changsha, Hunan 410011; \\ Departments of ${ }^{2}$ Geriatrics and ${ }^{3}$ Infectious Diseases, Hunan Provincial People's Hospital, Hunan Normal University, \\ Changsha, Hunan 410005; ${ }^{4}$ Department of Pharmacology, Xiangya School of Pharmaceutical Sciences, \\ Central South University, Changsha, Hunan 410078, P.R. China
}

Received May 17, 2018; Accepted October 10, 2018

DOI: $10.3892 /$ ijmm.2018.3965

\begin{abstract}
The present study aimed to determine whether isorhamnetin (Isor), a natural antioxidant polyphenol, has antifibrotic effects in a murine model of bleomycin-induced pulmonary fibrosis. A C57 mouse model of pulmonary fibrosis was established by intraperitoneal injection of a single dose of bleomycin $(3.5 \mathrm{U} / \mathrm{kg})$, and then Isor $(10$ and $30 \mathrm{mg} / \mathrm{kg}$ ) was administered intragastrically. The level of fibrosis was assessed by hematoxylin and eosin and Sirius red staining. $\alpha$-smooth muscle actin and type I collagen levels in lung tissues were determined by western blotting and immunohistochemistry (IHC). Epithelial-mesenchymal transition (EMT), endoplasmic reticulum stress (ERS) and related signaling pathways were examined by western blotting and IHC. In vitro, human bronchial epithelial cells (HBECs) and A549 cells were treated with transforming growth factor (TGF) $\beta 1$ with or without Isor, and collagen deposition and the expression levels of EMT- and ERS-related genes or proteins were analyzed by reverse transcription-quantitative polymerase chain reaction, western blotting, and immunofluorescence. The results demonstrated that Isor inhibited bleomycin-induced collagen deposition, reduced type I collagen and $\alpha$-SMA expression, and alleviated EMT and ERS in vivo. Furthermore, incubation of HBECs and A549 cells with TGF $\beta 1$ activated EMT and ERS, and this effect was reversed by Isor. In conclusion, Isor treatment attenuated bleomycin-induced EMT and pulmonary
\end{abstract}

Correspondence to: Professor Yu Yang, Department of Geriatrics, The Second Xiangya Hospital of Central South University, 139 Middle Renmin Road, Changsha, Hunan 410011, P.R. China E-mail: yangyu3@csu.edu.cn

Key words: isorhamnetin, epithelial-mesenchymal transition, endoplasmic reticulum stress, bleomycin-induced pulmonary fibrosis, protein kinase R-like endoplasmic reticulum kinase, collagen deposition fibrosis and suppressed bleomycin-induced ERS and the activation of PERK signaling.

\section{Introduction}

Pulmonary fibrosis is a chronic and progressive disease characterized by alveolar epithelial injury and abnormal collagen production $(1,2)$. Patients with pulmonary fibrosis often exhibit these effects, and pulmonary function is irreversibly lost (3). Clinical research has shown that patients with pulmonary fibrosis have a median survival of only 2.5-3.5 years after diagnosis. Though the current treatment options (pirfenidone and nintedanib) have improved, the prognosis of pulmonary fibrosis has not improved (4,5). Therefore, safer drugs with improved efficacy are needed to treat pulmonary fibrosis.

Pulmonary fibrosis is caused by abnormal proliferation of myofibroblasts and fibroblasts, which secrete excessive extracellular matrix (ECM) proteins $(4,5)$. Epithelial-mesenchymal transition (EMT) is a process in which polarized immotile epithelial cells are converted to motile mesenchymal cells. This is characterized by the loss of proteins associated with the polarized epithelial phenotype, such as E-cadherin, and an increase in mesenchymal markers such as vimentin and fibronectin $(6,7)$. In addition, EMT has been reported to be involved in myofibroblast formation (8). During pulmonary fibrosis, alveolar epithelial cells (AECs) contribute to the formation of myofibroblasts through EMT (9). Thus, an approach targeting EMT might also be promising in the treatment of pulmonary fibrosis (10).

The endoplasmic reticulum (ER), an important organelle, serves as a key role in biological synthesis, including correct protein folding, secretion and membrane protein post-translational modifications (11). Endoplasmic reticulum stress (ERS) triggers the activation of the unfolded protein response (UPR). The UPR and the endoplasmic reticulum response have been recognized to be activated in response to injury stress, oxidative stress, inflammation, infection and gene mutations, and are involved in conditions such as chronic liver fibrosis (12), pulmonary fibrosis (13-16), renal fibrosis (17) and myocardial fibrosis $(18,19)$. 
Isorhamnetin (Isor; molecular formula, $\mathrm{C}_{16} \mathrm{H}_{12} \mathrm{O}_{7}$ ), a flavonol aglycone isolated from the plant Hippophae rhamnoides $\mathrm{L}$, is frequently used in traditional Chinese medicine to prevent and treat various diseases. Isor has been demonstrated to exert a variety of effects, including antioxidant, anti-inflammatory, antitumor, antiviral, anti-ERS and neurodegenerative injury protection effects $(20,21)$. Recent research has revealed that Isor attenuates liver fibrosis by inhibiting transforming growth factor (TGF) $\beta /$ SMAD signaling and relieving oxidative stress (22). Furthermore, Isor has been reported to alleviate lipopolysaccharide-induced acute lung injury in mice (23). Lung injury can lead to pulmonary fibrosis (24). Therefore, the present study speculated that Isor may have a key role in pulmonary fibrosis. However, the function and mechanism have not yet been clarified.

In the present study, the effect of Isor on bleomycin (BLM)-induced pulmonary fibrosis was investigated. The results demonstrated that Isor mitigated pulmonary fibrosis induced by BLM. Mechanistically, the results revealed that Isor-mediated ERS prevention was partially dependent on the regulation of EMT progression. Based on the present findings, Isor might serve as a potential therapeutic strategy for the treatment of pulmonary fibrosis.

\section{Materials and methods}

Reagents and antibodies. Recombinant human TGF $\beta 1$ was purchased from PeproTech, Inc. (Rocky Hill, NJ, USA). Isor was purchased from Baomanbio (Shanghai, China). BLM was purchased from Hisun Company (Zhejiang, China). Antibodies targeting collagen I (dilution, 1:6,000; cat. no. ab138492), $\alpha$-smooth muscle actin ( $\alpha$-SMA; dilution, 1:300; ab32575) and $78 \mathrm{kDa}$ glucose-regulating protein (GRP78)/binding immunoglobulin protein (BiP; dilution, 1:1,200; cat. no. BM0134) were obtained from Abcam (Cambridge, UK). Antibodies targeting TGF $\beta 1$ (dilution, 1:1,000; cat. no. AM4195), protein kinase R-like endoplasmic reticulum kinase (PERK; dilution, 1:1,200; cat. no. BM0524) and E-cadherin (dilution, 1:1,200; cat no. BM0537) were obtained from Abzoom Biolabs, Inc. (Dallas, TX, USA). Antibodies targeting vimentin (dilution, 1:1,200; YT4880), phosphorylated (p)-PERK (dilution, 1:2,000; cat. no. YP1055), DNA damage-inducible transcript 3 (DDIT3; also known as CHOP; dilution, 1:1,200; cat. no. YT0911), eukaryotic translation initiation factor 2 subunit $\alpha$ (eIF2 $\alpha$; dilution, 1:1,200; cat. no. YT1507) and p-eIF2 $\alpha$ (dilution, 1:1,000; cat. no. YP0093) were obtained from ImmunoWay Biotechnology, Plano, TX, USA. Horseradish peroxidase (HRP)-coupled sheep anti-rat (dilution, 1:15,000; cat. no. SA001) or sheep anti-rabbit (dilution, 1:15,000; cat. no. SA009) secondary antibodies were obtained from Auragene Technology, Co., Inc. (Changsha, China).

BLM-induced pulmonary fibrosis and treatment. A total of 15 male 4-week old C57 mice (20-25 g in weight; SLRC Laboratory Animal Company, Changsha, China) were housed in rooms with a 12 -h light/dark cycle at $25^{\circ} \mathrm{C}$ and $40-70 \%$ humidity for 1 week prior to the experiment. Mice were fasted for $12 \mathrm{~h}$ and had ad libitum access to fresh tap water up until the beginning of the experiment. During the experiment, the mice had ad libitum access to food and water. The mice were then randomly assigned to the Isor treatment group, the BLM group or the control group. Mice in the Isor and BLM groups were intraperitoneally injected with BLM (3.5 U/kg; Hisun Company, Zhejiang, China), while mice in the control group were injected with normal saline. The Isor treatment group was divided into two subgroups: High dose $(30 \mathrm{mg} / \mathrm{kg})$ and low dose $(10 \mathrm{mg} / \mathrm{kg})$. Each subgroup was treated with Isor by intragastric administration once a day. Mice in the control group and BLM group were administered the same volume of distilled water by gavage. After 28 days, the mice were euthanized by pentobarbitone overdose. Lung tissues were collected and used for hematoxylin and eosin (H\&E) and Sirius red staining and western blot analyses. All experiments involving animals were approved by the Ethics Committee of Hunan Normal University Medical College (Changsha, China).

Cell culture. Human A549 cells and human bronchial epithelial cells (HBECs) were obtained from the Type Culture Collection of the Chinese Academy of Sciences (Shanghai, China) and cultured in Dulbecco's modified Eagle's medium (DMEM) supplemented with $10 \%$ fetal bovine serum (Thermo Fisher Scientific, Inc., Waltham, MA, USA) at $37^{\circ} \mathrm{C}$ with $5 \% \mathrm{CO}_{2}$. These cells were divided into five groups: The control group, the TGF $\beta 1$ group, and three TGF $\beta 1+$ Isor groups. Cells in the TGF $\beta 1$ group were stimulated with TGF $\beta 1$ alone $(5 \mathrm{ng} / \mathrm{ml})$ for $48 \mathrm{~h}$. Cells in the TGF $\beta 1+$ Isor groups were treated with $5 \mathrm{ng} / \mathrm{ml} \mathrm{TGF} \beta 1$ and 25,50 or $100 \mu \mathrm{M}$ Isor for $48 \mathrm{~h}$.

Histological and immunohistochemical analyses. The lung specimens were fixed in $4 \%$ paraformaldehyde at room temperature for $48 \mathrm{~h}$, dehydrated in a graded alcohol series and embedded in paraffin blocks. Sections were blocked with $3 \%$ hydrogen peroxide for $15 \mathrm{~min}$ at room temperature. Five-micron-thick sections were then stained with H\&E for $10 \mathrm{~min}$ for routine examination, or with Sirius red for $8 \mathrm{~min}$ to visualize collagen deposition at room temperature. For immunohistochemistry staining, sections were stained with the aforementioned antibodies, developed with 3,3'-diaminobenzidine, and counterstained with hematoxylin. Samples were viewed using a light microscope (magnification for $\mathrm{H} \& \mathrm{E}$ staining, x100 and x400; magnification for immunohistochemistry staining, $\mathrm{x} 200$ and $\mathrm{x} 400$ ).

Reverse transcription-quantitative polymerase chain reaction $(R T-q P C R)$. Total RNA was extracted with TRIzol reagent (Thermo Fisher Scientific, Inc.). A total of $2 \mu \mathrm{g}$ RNA was subjected to reverse transcription using the PrimeScript Reverse Transcription Reagent kit (Thermo Fisher Scientific, Inc.). Quantitative analysis of the change in expression levels was performed using SYBR Premix Ex Taq (Dongsheng Biotech, Guangzhou, China) on an ABI 7300 system (Applied Biosystems; Thermo Fisher Scientific, Inc.). The thermocycling conditions were as follows: An initial incubation at $95^{\circ} \mathrm{C}$ for $10 \mathrm{sec}$, followed by 40 cycles of denaturation at $95^{\circ} \mathrm{C}$ for $10 \mathrm{sec}$ and annealing at $60^{\circ} \mathrm{C}$ for $30 \mathrm{sec}$. The sequences of the primers used were: GRP78, sense, 5'-GAAGGTTACCCATGCA GTT-3' and antisense, 5'-AGCAATAGTTCCAGCGTCT-3'; CHOP, sense, 5'-CCTGAAAGCAGCACCAAA-3' and antisense, 5'-ACAAGCTCCATGTAGCAAAC-3'; and $\beta$-actin, sense, 5'-AGGGGCCGGACTCGTCATACT-3' and antisense, 
5'-GGCGGCACCACCATGTACCCT-3'. Data analysis was performed by the comparative $\mathrm{C}_{\mathrm{q}}$ method using $\mathrm{ABI}$ software (25). mRNA expression was normalized to that of $\beta$-actin.

Western blot analysis. Protein was extracted from lung tissues and cells with RIPA lysis buffer (Auragene Technology, Co., Inc., Shenzen, China). Protein concentrations were determined with bicinchoninic acid protein assay reagents (Auragene Technology, Co., Inc.) according to the manufacturer's instructions. Equal amounts of protein $(10 \mu \mathrm{g})$ from each sample were separated by $10 \%$ SDS-PAGE and transferred to polyvinylidene fluoride membranes. Membranes were blocked with $3 \%$ BSA-TBST at room temperature for $60 \mathrm{~min}$. The membranes were probed with primary antibodies overnight at $4^{\circ} \mathrm{C}$ and then incubated with a HRP-coupled sheep anti-rat (dilution, 1:15,000; cat. no. SA001) or sheep anti-rabbit (dilution, 1:15,000; cat. no. SA009; both from Auragene Technology, Co., Inc.) secondary antibody at room temperature for $40 \mathrm{~min}$. Signals were visualized with an enhanced chemiluminescence detection kit (Auragene Technology, Co., Inc.). Densitometric analysis was performed using Image-Pro Plus v. 6.0 (Media Cybernetics, Silver Spring, MD, USA).

Immunofluorescence cytochemistry. Cells were fixed in $4 \%$ paraformaldehyde for $30 \mathrm{~min}$ at room temperature. After three 5-min washes with PBS, the cells were blocked in PBS containing 3\% goat serum (OriGene Technologies, Inc., Beijing, China) and $0.5 \%$ Triton X-100 for $1 \mathrm{~h}$ at room temperature. Afterwards, the cells were incubated with the indicated primary antibodies at $4^{\circ} \mathrm{C}$ overnight and then incubated with Cy3-conjugated goat anti-rabbit $\operatorname{IgG}(\mathrm{H}+\mathrm{L})$ secondary antibody (1:500) for $1 \mathrm{~h}$ at room temperature (catalog no. SA012; Auragene Technology, Co., Inc.). The coverslips were counterstained with DAPI and imaged with a confocal laser scanning microscope (AE31; Motic Group, Co., Ltd., Xiamen, China).

Statistical analysis. Data from three independent experiments were expressed as the mean \pm standard deviation and processed using SPSS 17.0 statistical software (SPSS, Inc., Chicago, IL, USA). Differences between groups were evaluated by one-way analysis of variance followed by Tukey's test. $\mathrm{P}<0.05$ was considered to indicate a statistically significant difference.

\section{Results}

Isor alleviates BLM-induced pulmonary fibrosis in mice. In accordance with previous studies $(26,27)$, histological examination of lung tissues demonstrated that four weeks of exposure to bleomycin in mice led to significant pulmonary fibrotic changes, including disturbed alveolar structure, marked thickening of the interalveolar septa and dense interstitial infiltration by inflammatory cells and fibroblasts (Fig. 1A). However, Isor treatment (10 and $30 \mathrm{mg} / \mathrm{kg}$ ) significantly attenuated the fibrotic changes induced by BLM (Fig. 1A), and the higher concentration exhibited better inhibitory effect on fibrosis, with the histological morphology being similar to the control mice. The hallmark characteristic of BLM-induced pulmonary fibrosis is the excessive deposition of extracellular matrix proteins, such as collagen. Collagen deposition was clearly observed in the lungs of BLM-treated mice by Sirius red staining (Fig. 1B). As expected, Isor treatment significantly attenuated matrix protein deposition in the lungs (Fig. 1B). In addition, $\alpha$-SMA is a hallmark of myofibroblasts and a marker of pulmonary fibrosis. As illustrated in Fig. 1C, the protein expression levels of $\alpha$-SMA and collagen I were markedly upregulated in the lungs of BLM-treated mice. Isor treatment significantly reduced the production of collagen I and $\alpha$-SMA in BLM-treated lungs (Fig. 1C). The expression of $\alpha$-SMA was also monitored by immunohistochemical analysis. BLM treatment resulted in markedly increased expression of $\alpha$-SMA in the area of pulmonary fibrosis, while this effect was largely reversed in the Isor-treated groups (Fig. 1D).

Isor inhibits BLM-induced pulmonary ERS. Recent studies have demonstrated that pulmonary ERS is involved in the pathogenesis of BLM-induced pulmonary fibrosis (14-16). Therefore, the effects of Isor on BLM-induced pulmonary stress were analyzed. The protein expression levels of GRP78 and CHOP were examined, as both proteins are widely used as markers for ERS. As illustrated in Fig. 2A and B, the expression of GRP78 and CHOP was upregulated in the BLM-treated mice compared with the control mice, at both the protein and mRNA level. Isor treatment significantly attenuated the BLM-induced increase in GRP78 and CHOP expression, at both the transcriptional and translational level (Fig. 2A and B). These findings suggested that Isor treatment inhibited ERS, which was in accordance with a previous study (28). Immunohistochemistry staining for CHOP was performed in lung tissues for further confirmation. CHOP expression was decreased in Isor-treated BLM-induced lung tissues (10 and $30 \mathrm{mg} / \mathrm{kg}$ ) compared with BLM-induced lung tissues (Fig. 2C).

Isor alleviates ERS-mediated EMT. ERS-induced EMT contributes to fibrotic remodeling in the lungs $(15,29)$. Isor obviously inhibited ERS-induced EMT of myofibroblasts in the lungs, as evidenced by repression of pulmonary $\alpha$-SMA expression described above. For further confirmation, expression of the EMT-related proteins E-cadherin, Vimentin and TGF $\beta 1$ was evaluated by western blotting. The results demonstrated a significant upregulation of Vimentin and TGF $\beta 1$ expression and downregulation of E-cadherin expression in the BLM-treated group compared with the control group (Fig. 3A-E). However, Isor treatment alleviated the increase in Vimentin and TGF $\beta 1$ expression and the decrease in E-cadherin expression, compared with the BLM-treated group (Fig. 3A-E). These findings indicated that Isor treatment alleviated ERS-mediated EMT in BLM-induced pulmonary fibrosis in vivo.

Isor inhibits ERS by downregulating p-PERK. To investigate the possible pathways by which Isor inhibits ERS, the expression levels of total and phosphorylated PERK were determined by western blotting. PERK phosphorylation levels significantly increased in the BLM-induced group compared with the control group, while a dramatic decrease in p-PERK was observed in the Isor-treated BLM groups (Fig. 4A). Immunohistochemistry staining results for p-PERK in lung tissue sections supported the western blot analysis results (Fig. 4B). eIF2 $\alpha$ is a downstream target of the PERK 
A

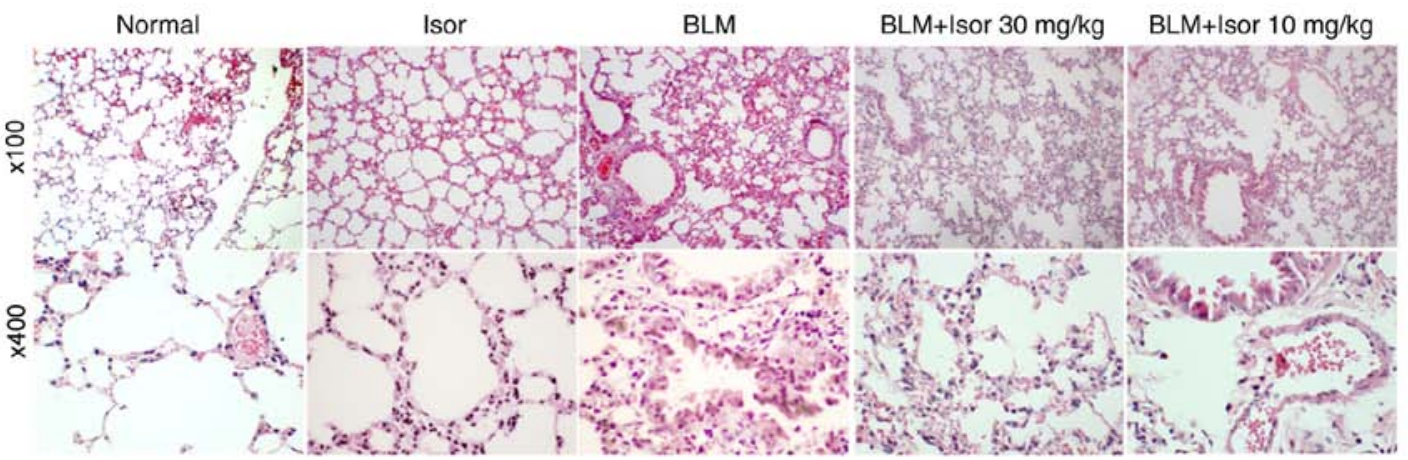

B

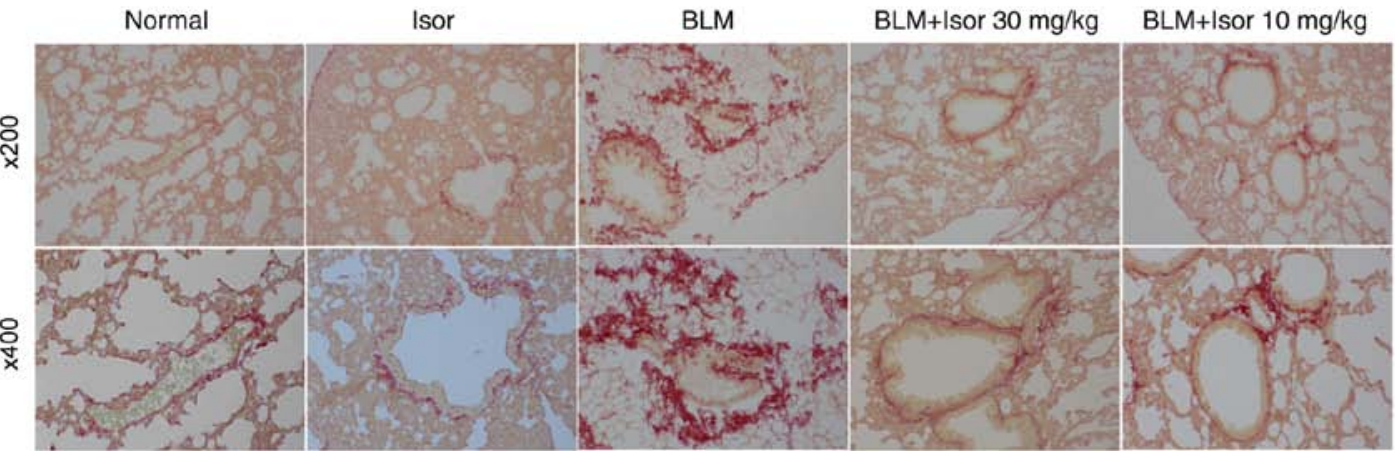

$\mathrm{C}$
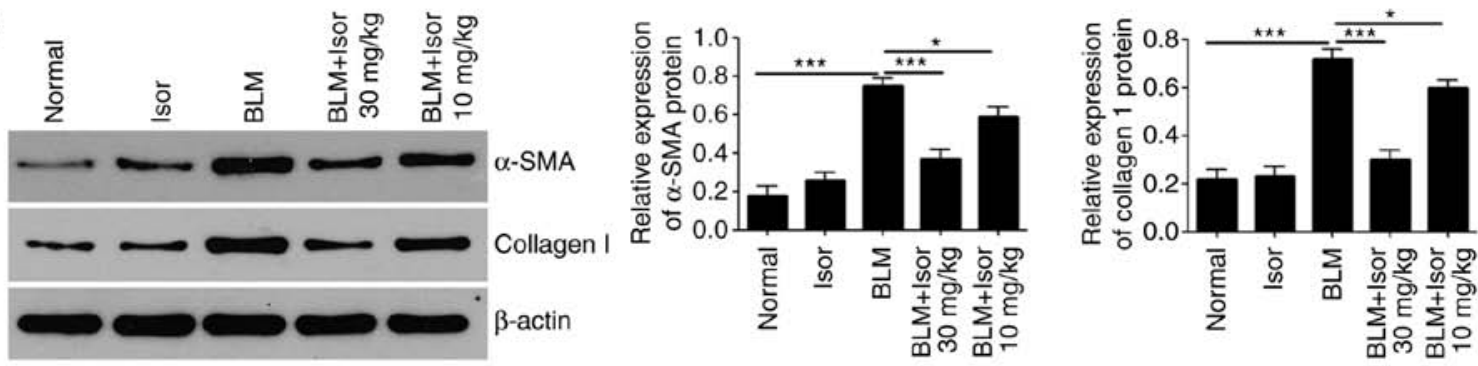

D

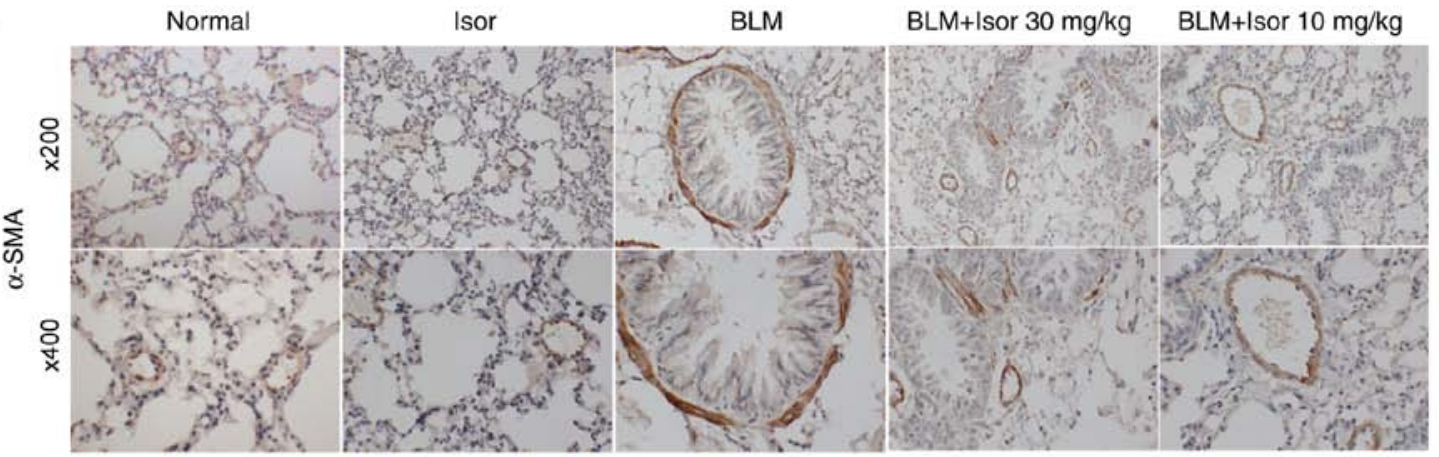

Figure 1. Effects of Isor on BLM-induced pulmonary fibrosis. (A) Representative images from hematoxylin and eosin staining showing lung pathologic abnormalities (magnification, x100 and x400). (B) Representative images from Sirius red staining showing collagen deposition (magnification, x200 and x400). (C) The protein expression levels of $\alpha$-SMA and collagen I were assessed by western blot analysis, and the densitometry values were normalized to $\beta$-actin. (D) Immunohistochemistry staining of $\alpha$-SMA in lung tissue sections (magnification, $\mathrm{x} 200$ and $\mathrm{x} 400$ ). Data are presented as the mean \pm standard deviation $(\mathrm{n}=3) .{ }^{*} \mathrm{P}<0.05$ and ${ }^{* * *} \mathrm{P}<0.001$, with comparisons indicated by lines. Isor, isorhamnetin; BLM, bleomycin; $\alpha$-SMA, $\alpha$-smooth muscle actin.

pathway. As illustrated in Fig. 4A, consistent with PERK phosphorylation, the levels of phosphorylated eIF2 $\alpha$ in the lungs of BLM-treated mice were elevated dramatically. Isor treatment significantly attenuated BLM-induced eIF $2 \alpha$ phosphorylation in the lungs (Fig. 4A). Thus, Isor exhibited an antifibrotic effect on BLM-induced pulmonary fibrosis in vivo.

Effects of Isor on the expression of fibrosis-associated factors and ERS markers in A549 cells and HBECs induced by TGF $\beta 1$.
To further validate the antifibrotic effect of Isor, in vitro studies were conducted in A549 cells and HBECs simulated with TGF $\beta 1$. Results obtained in vitro were similar to those observed in vivo. Treatment of cells with TGF $\beta 1$ increased the expression of fibrosis-related factors (collagen I and $\alpha$-SMA; Fig. 5) and ER markers (GRP78 and CHOP; Fig. 6). However, Isor suppressed the upregulated expression of collagen I, $\alpha$-SMA, GRP78 and CHOP in the presence of TGF $\beta 1$ in a dose-dependent manner (Figs. 5 and 6). 

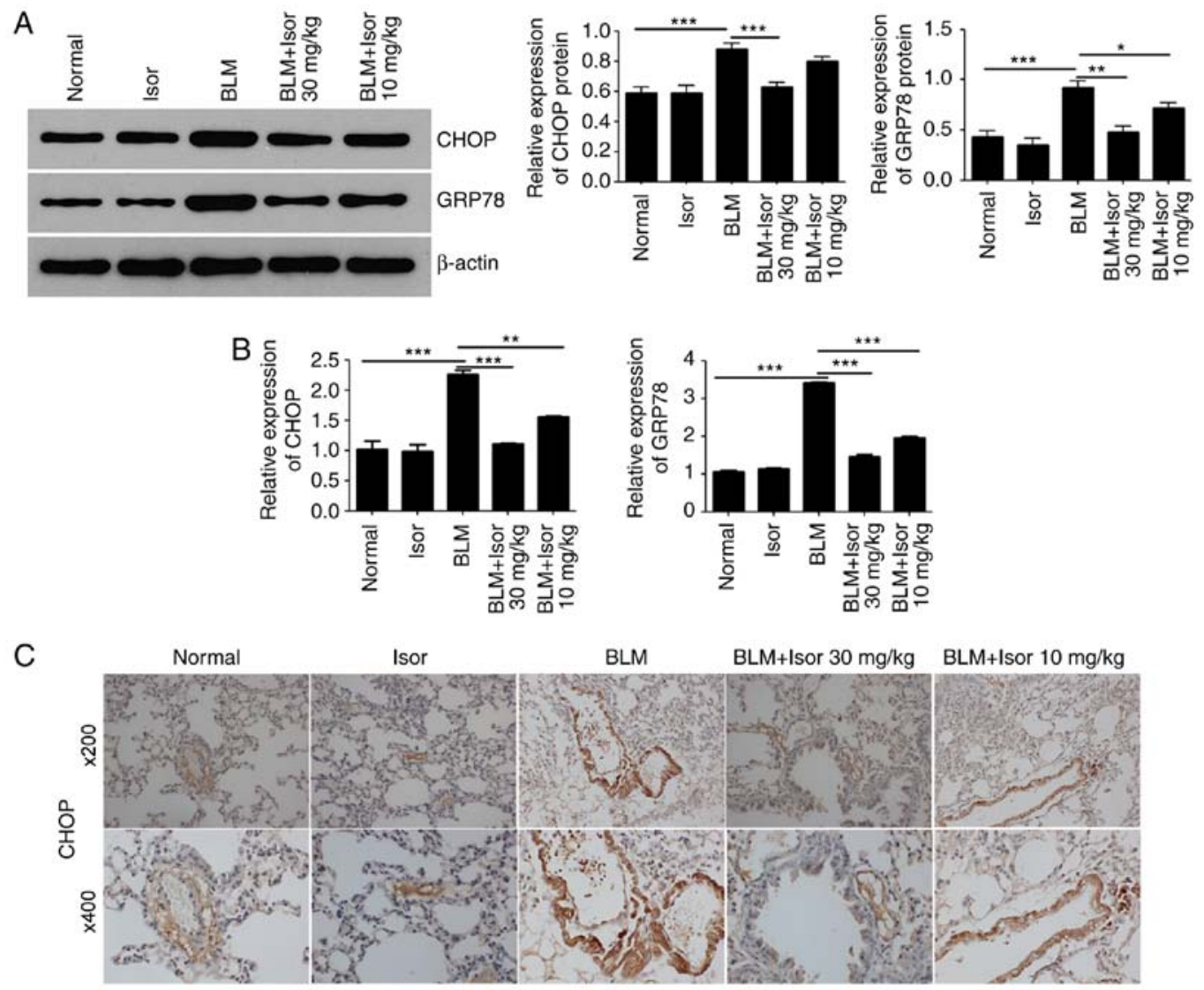

Figure 2. Effects of Isor on BLM-induced pulmonary endoplasmic reticulum stress markers. (A) The protein expression levels of CHOP and GRP78 were assessed by western blot analysis, and the densitometry values were normalized to $\beta$-actin. (B) The mRNA expression levels of CHOP and GRP78 were determined by reverse transcription-quantitative polymerase chain reaction. (C) Immunohistochemistry staining of CHOP in lung tissue sections (magnification, $\mathrm{x} 200$ and $\mathrm{x} 400)$. Data are presented as the mean \pm standard deviation $(\mathrm{n}=3) .{ }^{*} \mathrm{P}<0.05,{ }^{* *} \mathrm{P}<0.01$ and ${ }^{* * *} \mathrm{P}<0.001$, with comparisons indicated by lines Isor, isorhamnetin; BLM, bleomycin; CHOP, DNA damage-inducible transcript 3; GRP78, 78 kDa glucose-regulating protein.
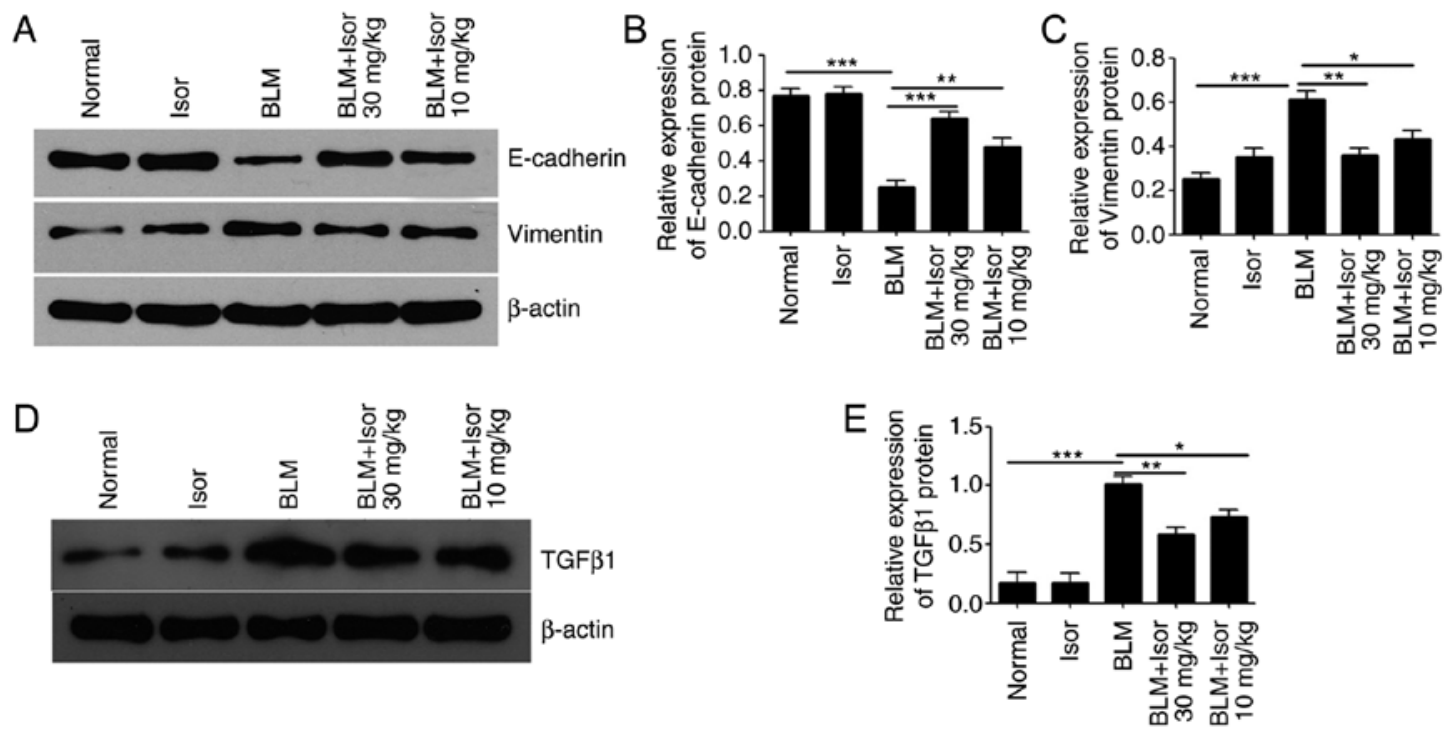

Figure 3. Effects of Isor on endoplasmic reticulum stress-mediated epithelial-mesenchymal transition. (A) The protein expression levels of E-cadherin and Vimentin, were evaluated by western blot analysis, and the densitometry values were normalized to $\beta$-actin. (B) Quantitative statistical analysis of E-cadherin. (C) Quantitative statistical analysis of Vimentin. (D) The protein expression levels of TGF $\beta 1$ in lung tissue sections was detected by western blot analysis. (E) Quantitative statistical analysis of TGF $\beta 1$. Data are presented as the mean \pm standard deviation $(\mathrm{n}=3) .{ }^{*} \mathrm{P}<0.05,{ }^{* * *} \mathrm{P}<0.01$ and ${ }^{* * *} \mathrm{P}<0.001$, with comparisons indicated by lines. Isor, isorhamnetin; TGF $\beta 1$, transforming growth factor $\beta 1$.

Effects of Isor on TGF 1 1-induced EMT in A549 cells and HBECs. Exposure to TGF $\beta 1$ caused A549 cells and HBECs to undergo EMT, during which the morphology of A549 cells changed from polygonal or cobblestone-like to a spindle-like 

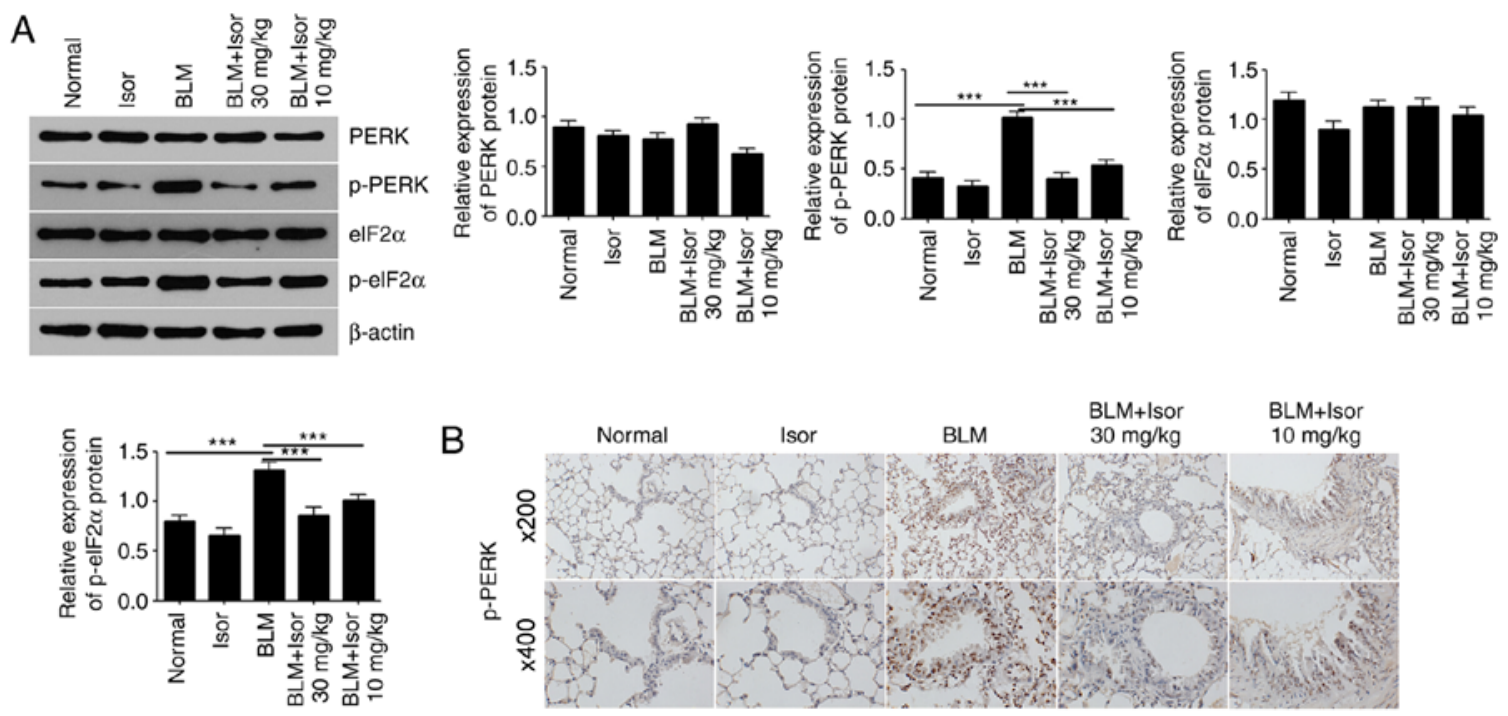

Figure 4. Effects of Isor on the expression of PERK and eIF2 $\alpha$. (A) The protein expression levels of total and phosphorylated PERK and eIF2 $\alpha$ were examined by western blot analysis, and the densitometry values were normalized to $\beta$-actin. (B) Immunohistochemical staining of p-PERK in lung tissue sections (magnification, $\mathrm{x} 200$ and $\mathrm{x} 400)$. Data are presented as the mean \pm standard deviation $(\mathrm{n}=3) .{ }^{* * *} \mathrm{P}<0.001$, with comparisons indicated by lines. Isor, isorhamnetin; PERK, protein kinase R-like endoplasmic reticulum kinase; eIF2 $\alpha$, eukaryotic translation initiation factor 2 subunit $\alpha$; p-, phosphorylated.
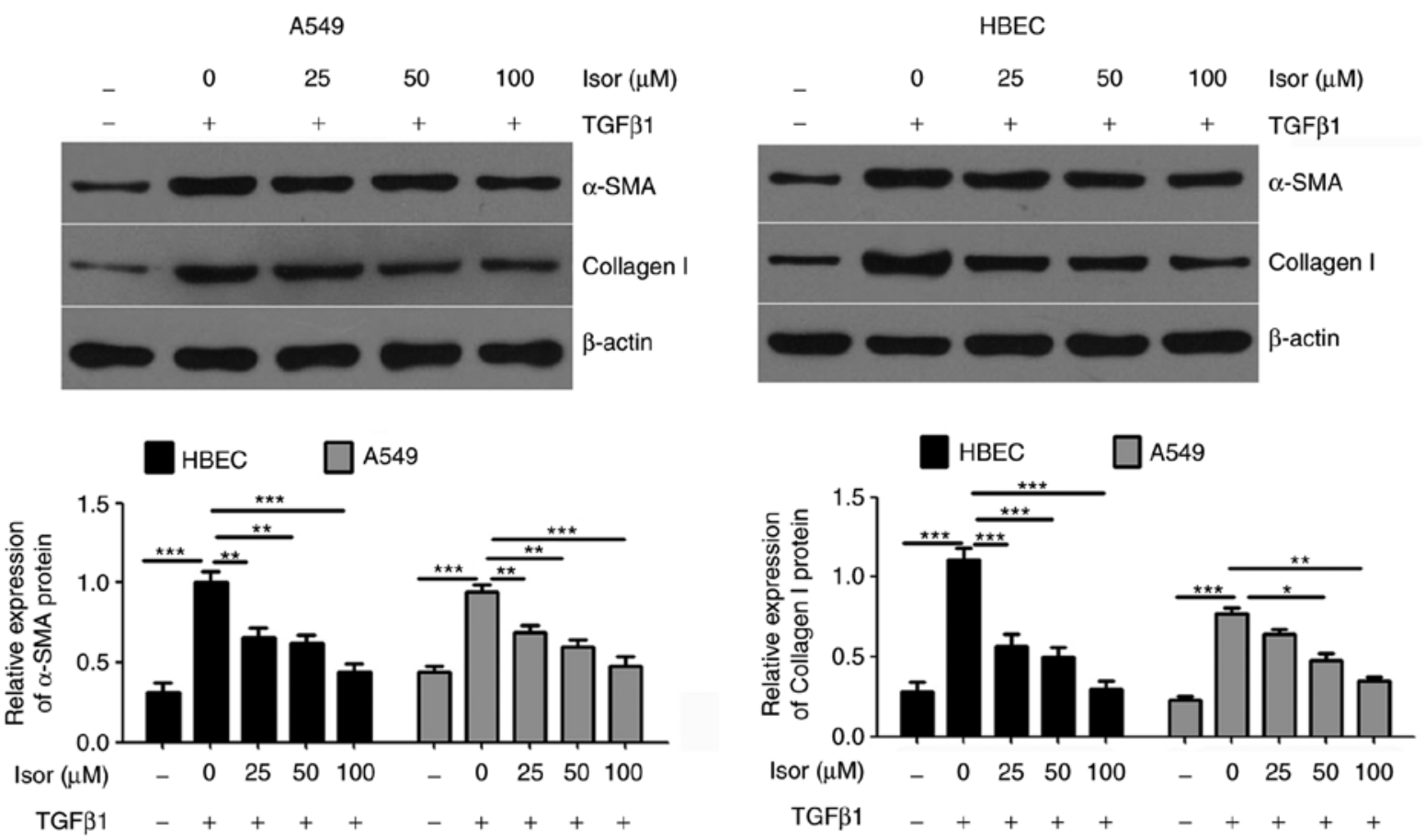

Figure 5. Effects of Isor on the expression of fibrosis-related factors in TGF $\beta 1$-induced A549 cells and HBECs. The protein expression levels of $\alpha$-SMA and collagen I were assessed by western blot analysis in A549 cells and in HBECs, and the densitometry values were normalized to $\beta$-actin. Data are presented as the mean \pm standard deviation $(\mathrm{n}=3)$. ${ }^{*} \mathrm{P}<0.05,{ }^{* *} \mathrm{P}<0.01$ and ${ }^{* * *} \mathrm{P}<0.001$, with comparisons indicated by lines. Isor, isorhamnetin; TGF $\beta 1$, transforming growth factor $\beta 1$; HBECs, human bronchial epithelial cells; $\alpha$-SMA, $\alpha$-smooth muscle actin.

shape, and that of HBECs changed from a short fusiform shape to a long fusiform shape (Fig. 7A). Aside from the morphological changes, the expression levels of E-cadherin were decreased, while the expression levels of Vimentin were increased (Fig. 7B); these results were also confirmed by immunostaining analysis (Fig. 7C). By contrast, treating cells with Isor reversed the TGF $\beta 1$-induced EMT in a dose-dependent manner, as illustrated by phenotypic cellular alterations (Fig. 7C) and the expression profiles of the EMT markers E-cadherin and
Vimentin (Fig. 7B). Taken together, the in vivo and in vitro data suggest that Isor protects against BLM-induced pulmonary fibrosis by inhibiting ERS-mediated EMT in the lungs.

\section{Discussion}

The present study demonstrated for the first time that Isor could inhibit pulmonary fibrosis in vivo and in vitro. The results also demonstrated that BLM and TGF $\beta 1$ upregulated EMT and 

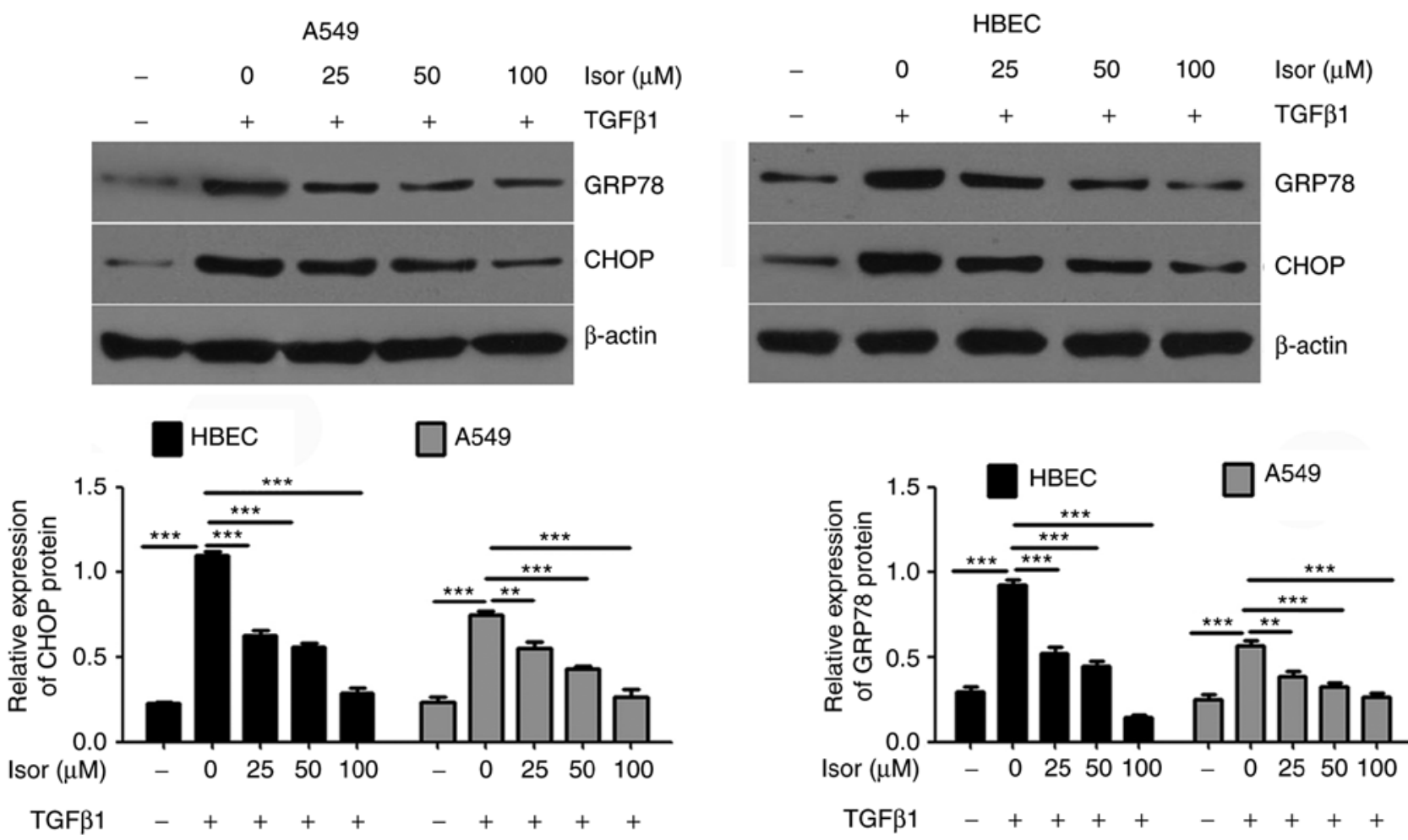

Figure 6. Effects of Isor on the expression of endoplasmic reticulum stress markers in TGFß1-induced A549 cells and HBECs. The protein expression levels of CHOP and GRP78 were assessed by western blot analysis in A549 cells and in HBECs, and the densitometry values were normalized to $\beta$-actin. Data are presented as the mean \pm standard deviation $(\mathrm{n}=3) .{ }^{* *} \mathrm{P}<0.01$ and ${ }^{* * *} \mathrm{P}<0.001$, with comparisons indicated by lines. Isor, isorhamnetin; TGF $\beta 1$, transforming growth factor $\beta 1$; HBECs, human bronchial epithelial cells; CHOP, DNA damage-inducible transcript 3; GRP78, 78 kDa glucose-regulating protein.

ERS in mice with pulmonary fibrosis and in cells, respectively. Isor, a natural antioxidant polyphenol, was capable of attenuating the effect of BLM-induced EMT and ERS in vivo and in vitro. In addition, it suppressed BLM-induced ERS by suppressing the PERK signaling pathway.

Increasing evidence has shown that EMT is involved in the formation of myofibroblasts and serves an important role in the pathogenesis of pulmonary fibrosis. In addition, inhibition of EMT could prevent epithelial cells from transforming into myofibroblasts, alleviating pulmonary fibrosis. For example, NLR family pyrin domain containing 3 (NLRP3) modulates EMT through the TGF $\beta 1$ signaling pathway, inhibiting pulmonary fibrosis (30). GHK inhibits BLM-induced fibrosis progression, the inflammatory response and EMT via the TGF $\beta 1 /$ Smad2/3 and insulin-like growth factor-1 (IGF-1) pathway (31). In the present study, the results demonstrated that Isor has the same function as NLRP3 and GHK, as it inhibited BLM- and TGF $\beta 1$-induced upregulation of $\mathrm{N}$-cadherin and downregulation of E-cadherin in mice and cells. Taken together, these results revealed that Isor inhibits BLM-induced pulmonary fibrosis through its inhibition of EMT in the lungs.

Multiple studies have confirmed that ERS and UPR signaling activation are involved in the pathogenesis of idiopathic pulmonary fibrosis, which includes elevated expression of GRP78, activating transcription factor 6 (ATF6), PERK, and inositol-requiring enzyme-1 (IRE-1) $(15,32)$. Previous studies have demonstrated that silencing of synoviolin and calcium/calmodulin-dependent protein kinase I (CaMKI) suppress ERS and alveolar epithelial cell apoptosis, protecting against pulmonary fibrosis (33), which indicates that ERS has an important role in pulmonary fibrosis. Wang et al (34) also reported that chlorogenic acid could inhibit pulmonary fibrosis through inhibition of ERS in vivo and in vitro. These previous findings support the current results. The present study investigated the effect of Isor on ERS, which was promoted by BLM-induced pulmonary fibrosis. The results demonstrated that the expression of ER chaperones (CHOP and GRP78) and members of the ERS signaling pathway (p-PERK and p-eIF $2 \alpha$ ) was markedly increased in mice with BLM-induced pulmonary fibrosis, and this effect was reversed by Isor treatment. Furthermore, it was demonstrated that Isor inhibited the ERS induced by TGF $\beta 1$ in A549 cells and HBECs in vitro. In addition, it is reported that Isor can inhibit ERS in obesity (27), and Isor inhibited ERS-induced injury through protein kinase $\mathrm{C}$ in $\mathrm{N} 2 \mathrm{a}$ cells, which is in accordance with the present results. Taken together, these results suggest that Isor inhibited BLM-induced pulmonary fibrosis through inhibition of ERS in the lung.

According to recent reports, ERS can induce EMT and the progression of pulmonary fibrosis. Inhibition of ERS could alleviate pulmonary fibrosis $(7,8)$. For example, melatonin inhibits ERS and EMT in BLM-induced pulmonary fibrosis (35). Therefore, Isor may inhibit EMT in pulmonary fibrosis via its inhibition of ERS in the lung. The target proteins involved in this process require further investigation.

In summary, the present study revealed that Isor inhibited EMT and ERS in BLM-induced pulmonary fibrosis via the PERK pathway. These findings may provide novel insights into the pathogenesis of pulmonary fibrosis. Additional 
A

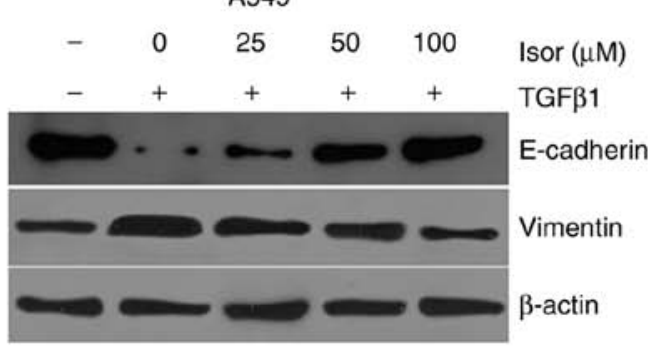

B

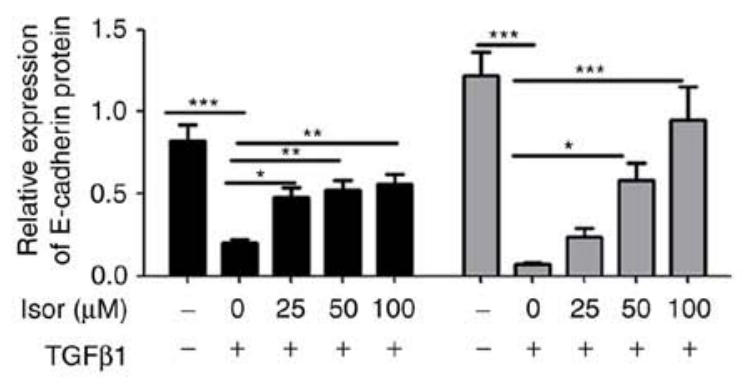

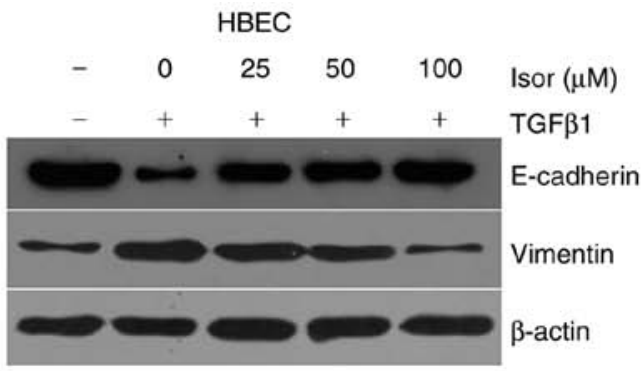

HBEC

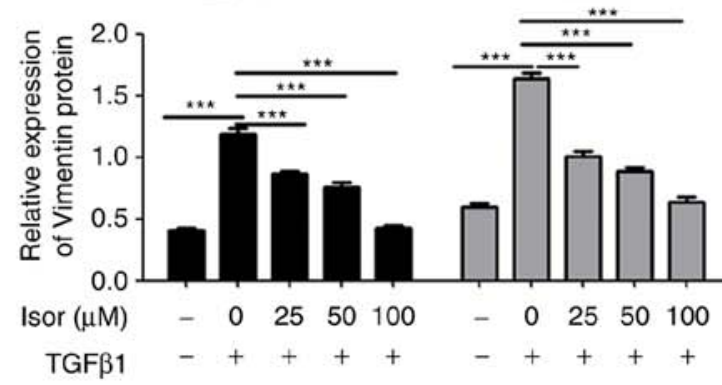

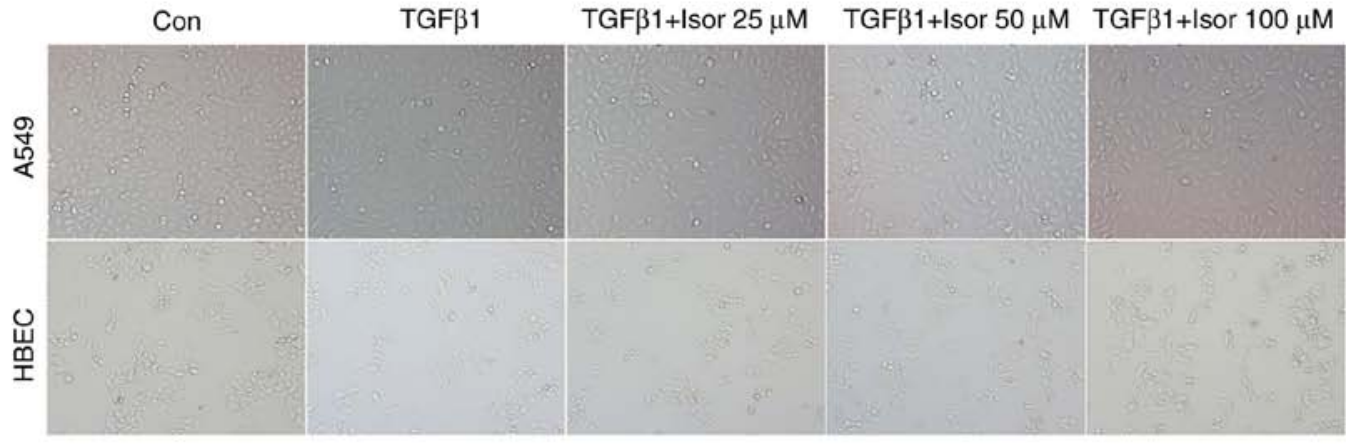

C
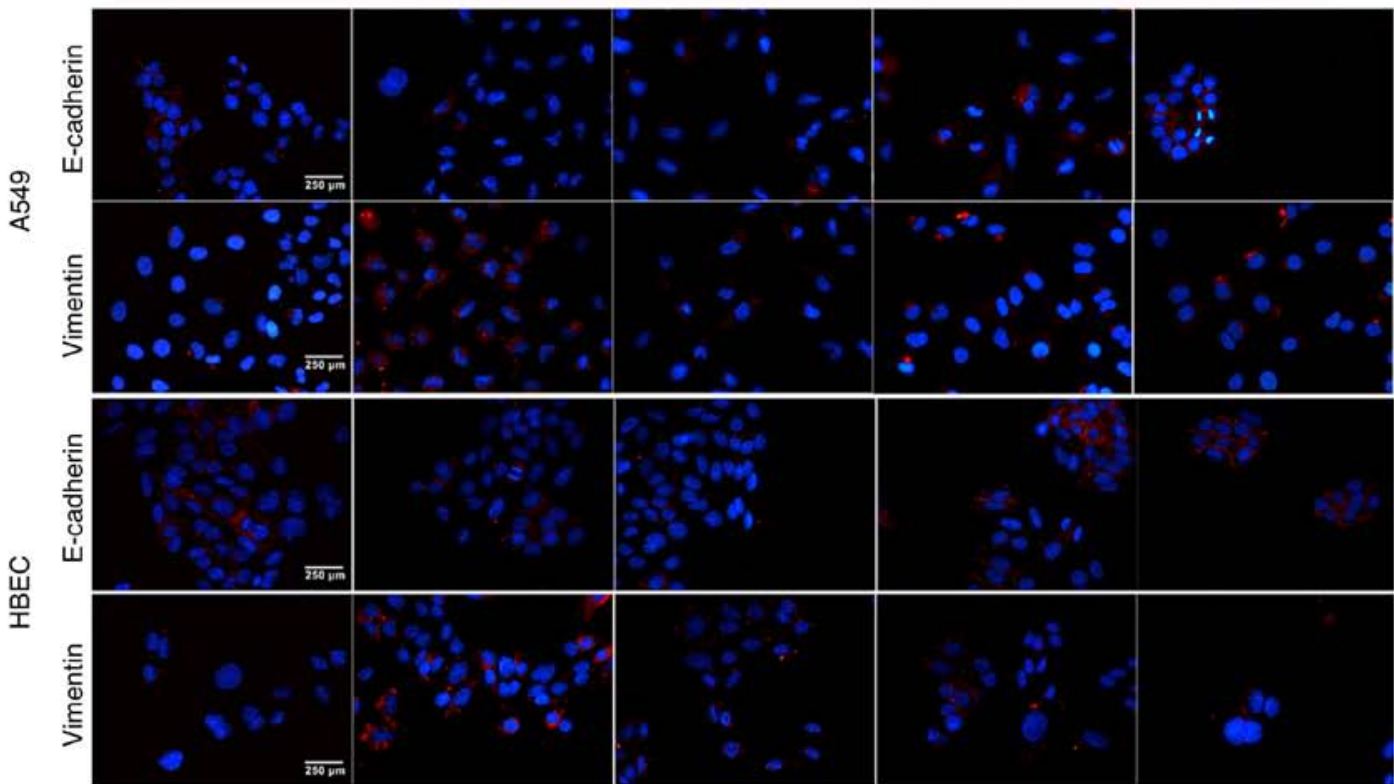

Figure 7. Effects of Isor on TGFß1-induced epithelial-mesenchymal transition in A549 cells and HBECs. (A) Morphological changes in Isor-treated A549 cells and HBECs in the presence of TGF $\beta 1$ were observed by phase contrast microscopy (magnification, $\mathrm{x} 200$ ). (B) The protein expression levels of E-cadherin and Vimentin were assessed by western blot analysis, and the densitometry values were normalized to $\beta$-actin. (C) Immunofluorescent staining for E-cadherin and Vimentin in Isor-treated A549 cells and HBECs in the presence of TGF $\beta 1$ (magnification, $\mathrm{x} 400)$. Data are presented as the mean \pm standard deviation $(\mathrm{n}=3)$. ${ }^{*} \mathrm{P}<0.05,{ }^{* * *} \mathrm{P}<0.01$ and ${ }^{* * *} \mathrm{P}<0.001$, with comparisons indicated by lines. Isor, isorhamnetin; TGF $\beta 1$, transforming growth factor $\beta 1$; HBECs, human bronchial epithelial cells.

investigations are necessary to elucidate the full antifibrotic potential of Isor as a concomitant therapy for patients with lung fibrosis, including that produced during BLM treatment, especially with regard to timing of its administration. 


\section{Acknowledgements}

Not applicable.

\section{Funding}

The present study was supported by the Science and Technology Innovation Program of Hunan Province (grant no. 2017SK50515).

\section{Availability of data and materials}

The analyzed datasets generated during the study are available from the corresponding author on reasonable request.

\section{Authors' contributions}

YY designed the study and obtained funding. QZ performed the animal experiments and tests. QZ, MT, BO, CL and $\mathrm{CH}$ performed the cell function assay. MT and YY provided major contributions to the writing of the manuscript. All authors read and approved the final manuscript.

\section{Ethics approval and consent to participate}

All experiments involving animals were approved by the Ethics Committee of Hunan Normal University Medical College (Changsha, China).

\section{Patient consent for publication}

Not applicable.

\section{Competing interests}

The authors declare that they have no competing interests.

\section{References}

1. King TE Jr, Pardo A and Selman M: Idiopathic pulmonary fibrosis. Lancet 378: 1949-1961, 2011.

2. Wolters PJ, Collard HR and Jones KD: Pathogenesis of idiopathic pulmonary fibrosis. Annu Rev Pathol 9: 157-179, 2014.

3. Raghu G, Collard HR, Egan JJ, Martinez FJ, Behr J, Brown KK Colby TV, Cordier JF, Flaherty KR, Lasky JA, et al: An official ATS/ERS/JRS/ALAT statement: Idiopathic pulmonary fibrosis: Evidence-based guidelines for diagnosis and management. Am J Respir Crit Care Med 183: 788-824, 2011.

4. Raghu G, Rochwerg B, Zhang Y, Garcia CA, Azuma A, Behr J, Brozek JL, Collard HR, Cunningham W, Homma S, et al: An official ATS/ERS/JRS/ALAT clinical practice guideline: Treatment of idiopathic pulmonary fibrosis. An update of the 2011 clinical practice guideline. Am J Respir Crit Care Med 192: e3-e19, 2015.

5. Walter N, Collard HR and King TE Jr: Current perspectives on the treatment of idiopathic pulmonary fibrosis. Proc Am Thorac Soc 3: 330-338, 2006.

6. Borensztajn K, Crestani B and Kolb M: Idiopathic pulmonary fibrosis: From epithelial injury to biomarkers-insights from the bench side. Respiration 86: 441-452, 2013.

7. Li T, Yang X, Xin S, Cao Y and Wang N: Paraquat poisoning induced pulmonary epithelial mesenchymal transition through Notch1 pathway. Sci Rep 7: 924, 2017.

8. Wang YC, Liu JS, Tang HK, Nie J, Zhu JX, Wen LL and Guo QL: miR-221 targets HMGA2 to inhibit bleomycin-induced pulmonary fibrosis by regulating TGF- $\beta 1 / \mathrm{Smad} 3$-induced EMT. Int J Mol Med 38: 1208-1216, 2016.
9. Huang M, Wang YP, Zhu LQ, Cai Q, Li H and Yang HF: MAPK pathway mediates epithelial-mesenchymal transition induced by paraquat in alveolar epithelial cells. Environ Toxicol 31: 1407-1414, 2016.

10. Takemasa A, Ishii Y and Fukuda T: A neutrophil elastase inhibitor prevents bleomycin-induced pulmonary fibrosis in mice. Eur Respir J 40: 1475-1482, 2012.

11. Back SH, Lee K, Vink E and Kaufman RJ: Cytoplasmic IRE1alpha-mediated XBP1 mRNA splicing in the absence of nuclear processing and endoplasmic reticulum stress. J Biol Chem 281: 18691-18706, 2006.

12. Galligan JJ, Smathers RL, Shearn CT, Fritz KS, Backos DS, Jiang H, Franklin CC, Orlicky DJ, Maclean KN and Petersen DR: Oxidative stress and the ER stress response in a murine model for early-stage alcoholic liver disease. J Toxicol 2012: 207594, 2012.

13. Baek HA, Kim DS, Park HS, Jang KY, Kang MJ, Lee DG, Moon WS, Chae HJ and Chung MJ: Involvement of endoplasmic reticulum stress in myofibroblastic differentiation of lung fibroblasts. Am J Respir Cell Mol Biol 46: 731-739, 2012.

14. Tanjore H, Lawson WE and Blackwell TS: Endoplasmic reticulum stress as a pro-fibrotic stimulus. Biochim Biophys Acta 1832: 940-947, 2013

15. Lawson WE, Cheng DS, Degryse AL, Tanjore H, Polosukhin VV, $\mathrm{Xu} \mathrm{XC}$, Newcomb DC, Jones BR, Roldan J, Lane KB, et al: Endoplasmic reticulum stress enhances fibrotic remodeling in the lungs. Proc Natl Acad Sci USA 108: 10562-10567, 2011.

16. Lawson WE, Crossno PF, Polosukhin VV, Roldan J, Cheng DS, Lane KB, Blackwell TR, Xu C, Markin C, Ware LB, et al: Endoplasmic reticulum stress in alveolar epithelial cells is prominent in IPF: Association with altered surfactant protein processing and herpesvirus infection. Am J Physiol Lung Cell Mol Physiol 294: L1119-L1126, 2008.

17. Chiang CK, Hsu SP, Wu CT, Huang JW, Cheng HT, Chang YW, Hung KY, Wu KD and Liu SH: Endoplasmic reticulum stress implicated in the development of renal fibrosis. Mol Med 17: 1295-1305, 2011.

18. Ayala P, Montenegro J, Vivar R, Letelier A, Urroz PA, Copaja M, Pivet D, Humeres C, Troncoso R, Vicencio JM, et al: Attenuation of endoplasmic reticulum stress using the chemical chaperone 4-phenylbutyric acid prevents cardiac fibrosis induced by isoproterenol. Exp Mol Pathol 92: 97-104, 2012.

19. Spitler KM and Webb RC: Endoplasmic reticulum stress contributes to aortic stiffening via proapoptotic and fibrotic signaling mechanisms. Hypertension 63: e40-e 45, 2014.

20. Ahn H and Lee GS: Isorhamnetin and hyperoside derived from water dropwort inhibits inflammasome activation. Phytomedicine 24: 77-86, 2017.

21. Gao L, Yao R, Liu Y, Wang Z, Huang Z, Du B, Zhang D, Wu L, Xiao $L$ and Zhang Y: Isorhamnetin protects against cardiac hypertrophy through blocking PI3K-AKT pathway. Mol Cell Biochem 429: 167-177, 2017.

22. Yang JH, Kim SC, Kim KM, Jang CH, Cho SS, Kim SJ, Ku SK, Cho IJ and Ki SH: Isorhamnetin attenuates liver fibrosis by inhibiting TGF- $\beta /$ Smad signaling and relieving oxidative stress. Eur J Pharmacol 783: 92-102, 2016.

23. Yang B, Li XP, Ni YF, Du HY, Wang R, Li MJ, Wang WC, Li MM, Wang XH, Li L, et al: Protective effect of isorhamnetin on lipopolysaccharide-induced acute lung injury in mice. Inflammation 39: 129-137, 2016.

24. Knudsen L, Ruppert C and Ochs M: Tissue remodelling in pulmonary fibrosis. Cell Tissue Res 367: 607-626, 2017.

25. Higashiyama H, Yoshimoto D, Okamoto Y, Kikkawa H, Asano S and Kinoshita M: Receptor-activated Smad localisation in bleomycin-induced pulmonary fibrosis. J Clin Pathol 60: 283-289, 2007.

26. Zhuang Y, Dai J, Wang Y, Zhang H, Li X, Wang C, Cao M, Liu Y, Ding J, Cai H, et al: MiR-338* targeting smoothened to inhibit pulmonary fibrosis by epithelial-mesenchymal transition. Am J Transl Res 8: 3206-3213, 2016.

27. Rodríguez-RodríguezC,TorresN,Gutiérrez-UribeJA,NoriegaLG, Torre-Villalvazo I, Leal-Díaz AM, Antunes-Ricardo M, Márquez-Mota C, Ordaz G, Chavez-Santoscoy RA, et al: The effect of isorhamnetin glycosides extracted from Opuntia ficus-indica in a mouse model of diet induced obesity. Food Funct 6: 805-815, 2015.

28. Zhao H, Qin HY, Cao LF, Chen YH, Tan ZX, Zhang C and Xu DX: Phenylbutyric acid inhibits epithelial-mesenchymal transition during bleomycin-induced lung fibrosis. Toxicol Lett 232: 213-220, 2015. 
29. Tian R,Zhu Y, Yao J, Meng X, Wang J, Xie H and Wang R: NLRP3 participates in the regulation of EMT in bleomycin-induced pulmonary fibrosis. Exp Cell Res 357: 328-334, 2017.

30. Zhou XM, Wang GL, Wang XB2, Liu L, Zhang Q, Yin Y, Wang QY, Kang J and Hou G: GHK peptide inhibits bleomycin-induced pulmonary fibrosis in mice by suppressing TGFbeta1/Smad-mediated epithelial-to-mesenchymal transition. Front Pharmacol 8: 904, 2017.

31. Wynn TA and Ramalingam TR: Mechanisms of fibrosis: Therapeutic translation for fibrotic disease. Nat Med 18: 1028-1040, 2012.

32. Uhal BD, Nguyen H, Dang M, Gopallawa I, Jiang J, Dang V, Ono $\mathrm{S}$ and Morimoto K: Abrogation of ER stress-induced apoptosis of alveolar epithelial cells by angiotensin 1-7. Am J Physiol Lung Cell Mol Physiol 305: L33-L41, 2013.

33. Winters CJ, Koval O, Murthy S, Allamargot C, Sebag SC, Paschke JD, Jaffer OA, Carter AB and Grumbach IM: CaMKII inhibition in type II pneumocytes protects from bleomycin-induced pulmonary fibrosis by preventing $\mathrm{Ca}^{2+}$-dependent apoptosis. Am J Physiol Lung Cell Mol Physiol 310: L86-L94, 2016.
34. Wang YC, Dong J, Nie J, Zhu JX, Wang H, Chen Q, Chen JY, Xia JM and Shuai W: Amelioration of bleomycin-induced pulmonary fibrosis by chlorogenic acid through endoplasmic reticulum stress inhibition. Apoptosis 22: 1147-1156, 2017.

35. PLOS ONE Staff: Correction: Melatonin inhibits endoplasmic reticulum stress and epithelial-mesenchymal transition during bleomycin-induced pulmonary fibrosis in mice. PLoS One 10: e119381, 2015.

This work is licensed under a Creative Commons Attribution-NonCommercial-NoDerivatives 4.0 International (CC BY-NC-ND 4.0) License. 